\title{
A importância do foco da terapia cognitivo-comportamental direcionado às sensações corporais no transtorno do pânico: relato de caso
}

\author{
The importance of the focus of the cognitive-behavior therapy related to the body \\ sensation in the panic disorder: case report
}

\author{
Anna Lucia Spear King ${ }^{1}$, Alexandre Martins Valença ${ }^{2}$, Valfrido Leão de Melo-Neto ${ }^{3}$, Antonio Egidio Nardi ${ }^{4}$ \\ - Psicóloga e mestranda do Programa de Pós-Graduação em Psiquiatria e Saúde Mental do Instituto de Psiquiatria da Universidade Federal do Rio de Janeiro (UFRJ). \\ 2 Professor adjunto de Psiquiatria do Centro de Ciências Médicas da Universidade Federal Fluminense (UFF) e pesquisador do Laboratório de Pânico e Respiração do IPUB (UFRJ). \\ ${ }^{3}$ Médico psiquiatra e mestrando do Programa de Pós-Graduação em Psiquiatria e Saúde Mental do Instituto de Psiquiatria da UFRJ.
}

${ }^{4}$ Livre-docente e professor adjunto do Instituto de Psiquiatria da UFRJ.

Recebido: 01/11/2006 - Aceito: 09/03/2007

\begin{abstract}
Resumo
Contexto: A conceituação cognitiva do transtorno de pânico (TP) realça os medos de sensações corporais (SC) em consequiência de avaliações distorcidas pelo indivíduo, que interpreta erroneamente as SC de forma catastrófica. Objetivo: A importância desse relato de caso é apresentar um trabalho intensivo de indução dos sintomas de ataque de pânico, com técnicas da terapia cognitivo-comportamental (TCC) relacionadas às SC. Método: O caso de E., feminino, 56 anos, foi retirado de uma pesquisa realizada no Laboratório de Pânico e Respiração do IPUB (UFRJ), com um grupo de pacientes com diagnóstico de TP que se tratou com medicação e sessões de TCC (16), com enfoque em exercícios de indução de sintomas, comparados com grupo controle que usou apenas medicação. Resultados: Os resultados foram controlados por questionários e escalas aplicados antes e após as intervenções. A paciente apresentava sintomas de hipocondria, queixas de falta de ar, taquicardia e medo de perder o controle, especialmente ao estar em ônibus, metrôs ou túneis. Ela recebeu prescrição do antidepressivo tricíclico, imipramina, $75 \mathrm{mg} /$ dia, e 16 sessões de TCC. Conclusão: Ao final, a paciente obteve remissão dos ataques de pânico e apresentou melhora significativa do comportamento agorafóbico.
\end{abstract}

King, A.L.S. et al. / Rev. Psiq. Clin 34(4); 191-195, 2007

Palavras-chave: Sensações corporais, terapia cognitivo-comportamental, ansiedade, diagnóstico, hipocondria.

\begin{abstract}
Background: The current cognitive conceptualization for the panic disorder (PD) enhances the fears of body sensations (BS) in consequence of evaluations distorted by the individual who interprets in an erroneous form the BS as catastrophic. Objective: The importance of this study is to emphasize the importance of an intensive work of induction of panic symptoms, with cognitive-behavioral therapy (CBT) techniques related to the BS. Method: The case of E., woman, 56 year-old, was selected from a research carried through in the Laboratory of Panic and Respiration - IPUB (UFRJ) with a group of patients with PD diagnosis treated with medication and 16 CBT sessions targeted in panic symptoms induction exercises, compared with a control group that used only medication. Results: The results had been controlled through questionnaires and scales applied before and after the interventions. The patient presented hypochondriac symptoms, sensation of shortness of breath, palpitations and fear or loosing control, especially when inside of buses, subways or tunnels. She received a tricycle antidepressant, imipramina, $75 \mathrm{mg} /$ day and $16 \mathrm{CBT}$ sessions. Conclusion: At the end of the trial, the patient had panic free status and presented significant improvement of the agoraphobic behavior.
\end{abstract}

King, A.L.S. et al. / Rev. Psiq. Clín 34(4); 191-195, 2007

Key-words: Body sensation, cognitive-behavior therapy, anxiety, diagnosis, hypochondriasis.

Endereço para correspondência: Anna Lucia Spear King. Rua Almirante Gomes Pereira, 8 - 22291-170 - Rio de Janeiro, RJ. Fone: (21) 2295-4477. E-mail: alking@unikey.com.br 


\section{Introdução}

Atualmente existe uma conceituação cognitiva específica para os ataques de pânico (AP) e o transtorno de pânico (TP) que realça os medos de sensações corporais (SC). Esse medo surge em consequiência de avaliações distorcidas pelo indivíduo, que interpreta as $\mathrm{SC}$ de forma catastrófica, como se fossem sinais de morte iminente e perda de controle, não conseguindo interpretá-las como reações fisiológicas naturais de adaptação provenientes do mecanismo biológico de "luta ou fuga" (Barlow, 1988; Clark et al., 1988; Ehlers e Margraf, 1989).

Diante de qualquer ameaça, situação estressante ou percebida como tal, o corpo reage com alterações fisiológicas, com o objetivo de criar condições físicas necessárias para enfrentar o problema ou fugir dele. Contudo, no TP, o indivíduo não percebe assim, assimilando equivocadamente os sintomas emergentes como algo grave. Em consequiência dessa cognição distorcida, o indivíduo pode inclusive desenvolver complicações do transtorno de pânico. Um exemplo é a hipocondria, transtorno caracterizado pela preocupação e medo excessivos de adquirir uma doença séria (Barsky et al., 1998).

O início dos sintomas de ataque de pânico ocorreria a partir do momento em que o indivíduo interpreta as sensações fisiológicas corporais normais emergentes como sintomas de doença grave ou morte. Quando essa associação distorcida se estabelece, passa a ser a referência cognitiva vigente do paciente (Barlow, 1988).

Os ataques de pânico (AP) podem apresentar maior tendência de se tornarem recorrentes quando existem associações entre certas sensações ou sintomas corporais como sinais de possíveis novos ataques. Sendo assim, as atividades que produzem SC semelhantes aos sintomas físicos de ataque de pânico, como exercícios físicos, estímulos sexuais, ingestão de cafeína, alterações térmicas, entre outras, costumam ativar os AP (Razran, 1961).

$\mathrm{O}$ ataque de pânico é uma ansiedade intensa, paroxística e intermitente. A ansiedade e sintomas fóbicos trazem sérias repercussões médicas, sociais e financeiras, associando-se a outros transtornos psiquiátricos, como abuso de substância, depressão e potencial para o suicídio (Markowitz et al., 1989; Rice e Miller, 1993; Wittchen, 1988; Zaubler e Katon, 1996). Em pesquisa realizada com 562 pacientes com TP ou agorafobia, ou ambos, acompanhados por 1 ano, a metade da amostra, tratada com TCC, apresentou melhora do quadro de ansiedade. A outra metade, tratada com terapia psicodinâmica, apresentou resultados inexpressivos (Goisman et al., 1994).

Apesar das sérias consequiências da ansiedade não tratada, entre 60\% e 92\% dos indivíduos afetados (Angst e Dobler-Mikola, 1985) não recebem tratamento, talvez em razão da falta de diagnóstico correto e maior custo da intervenção psicológica. Além disso, quando se obtém tratamento, não necessariamente é adequado e eficaz.
O foco da TCC nas SC no TP é fundamental na primeira etapa do tratamento, ou seja, nas primeiras oito sessões, para criar condições de o paciente reestruturar os aspectos cognitivos e mal-interpretados desenvolvidos que deram origem aos AP (Beck e Emery, 1985). $\mathrm{O}$ objetivo é fazer com que o indivíduo com TP perceba que as reações corporais emergentes são naturais, não representando perigo real, e derivadas de mecanismos fisiológicos. Sendo assim, é capaz de lidar com as SC sem medo, passando a elaborar novas estratégias de lidar com os pensamentos, sentimentos físicos e comportamentos até então distorcidos.

As técnicas utilizadas nas 16 sessões de TCC consistem na escuta das questões e queixas do paciente, na educação didática dele sobre os componentes do $\mathrm{TP}$ que incluem os conceitos de ansiedade, medo, pânico e hiperventilação. E, também, técnicas de respiração, relaxamento muscular e exercícios específicos e controlados de indução dos sintomas que expõem o paciente às próprias sensações físicas, com o objetivo de reconhecer que são inofensivas, podendo lidar com elas sem considerar que sejam o prenúncio de algo grave ou ameaçador.

Os exercícios de relaxamento muscular consistem em tencionar e depois relaxar por 1 minuto a musculatura de quatro grupamentos, rosto, tórax, pernas e braços concomitantes com a respiração, produzindo redução da tensão e ansiedade.

Os exercícios específicos e controlados de indução dos sintomas basearam-se em estudos anteriores de Lang e Craske (2003), tais como:

- inspirar e expirar profundamente (90 segundos);

- girar no lugar (1 minuto);

- virar a cabeça de um lado para o outro (30 segundos);

- colocar a cabeça entre as pernas (30 segundos) e levantar rapidamente;

- olhar fixo para uma luz (1 minuto) e depois tentar ler.

Com isso, a paciente pôde perceber que as sensações produzidas nos exercícios de indução dos sintomas, semelhantes aos dos ataques de pânico, eram inofensivas, não produziam as consequiências temidas e que teria meio de lidar com elas.

Em cada sessão, realizou-se apenas um exercício de indução de sintomas seguido por exercício de reeducação da respiração (inspirar contando até três), prender o ar (contando até três) e expirar (contando até seis), utilizando a musculatura diafragmática. Desde o início, o controle da hiperventilação foi feito com reeducação da respiração.

A reeducação da respiração para o TP é uma prática eficaz e produtiva, uma vez que entre $50 \%$ e $60 \%$ dos pacientes descrevem sintomas de hiperventilação como muito semelhantes aos sintomas de AP (Holt e Andrews, 1989). 
Elaboramos, ainda, uma hierarquia, traçada pelo próprio paciente, contendo seus medos menores e maiores a serem trabalhados na fase de exposição in vivo (expor o paciente gradativamente a situações agorafóbicas).

Enquanto não se obtiver sucesso na primeira fase do tratamento, não será possível passar para a etapa seguinte composta por mais oito sessões, de acordo com os métodos de pesquisa usados por Lang e Craske (2003), que consistem na exposição do indivíduo a situações agorafóbicas.

No tratamento com TCC, objetiva-se estimular o paciente a investigar as associações relacionadas às SC. Quando compreende os mecanismos do desencadeamento das SC, torna-se mais fácil desfazer e corrigir a cognição distorcida. A partir desse momento, o indivíduo restabelece o entendimento das sensações fisiológicas como quando fazia antes do TP, com bases reais e adaptativas.

\section{Relato de caso}

E., 56 anos, feminino, brasileira, natural do Rio de Janeiro, separada há 15 anos, duas filhas. Atualmente trabalha como esteticista e mora com a irmã e as filhas solteiras.

Procurou tratamento no Laboratório de Pânico e Respiração da UFRJ, em dezembro de 2005 , com queixas de ansiedade e medos, recebendo o diagnóstico de TP com agorafobia (DSM-IV-TR, 2000) e também após entrevista pela Structured Clinical Interview Diagnostic (SCID) for DSM-IV Axis I Disorders-Clinician Version (SCID-CV) (First et al., 1997). Relatava ter medo de utilizar metrô, ônibus cheio e passar por túneis longos e escuros.

A paciente apresentava TP do subtipo respiratório, estando esses sintomas respiratórios proeminentes na vigência do AP: falta de ar, sensação de sufocamento, dificuldade de respirar, tontura e palpitações. A conduta médica consistiu na prescrição do antidepressivo tricíclico, imipramina, $75 \mathrm{mg}$ ao dia, com reavaliação mensal e encaminhamento para TCC.

Este caso foi retirado da amostra de um estudo amplo realizado no Laboratório de Pânico e Respiração do IPUB (UFRJ), onde existiam dois grupos de pacientes com diagnóstico comum de TP com agorafobia e excluídas possíveis comorbidades. O primeiro grupo, tratado com o uso de medicação e TCC, foi comparado com um segundo grupo controle que apenas tomava medicação.

Com a finalidade de comparar os resultados dos tratamentos após as intervenções, aplicaram-se no início e ao final o questionário de medos e fobias (Marks e Mathews, 1979), o questionário de sensações corporais (Chambless et al., 1984), a escala de cognições agorafóbicas (Chambless et al., 1984), a escala para pânico e agorafobia (Bandelow, 1992), o inventário de mobilidade (Chambless et al., 1984) e o Beck Anxiety Inventory (Beck et al., 1988).
Após a primeira entrevista com a psicóloga para a coleta de dados e história pessoal, programaram-se, inicialmente, 16 sessões individuais de TCC com 1 hora de duração cada uma. A primeira fase da terapia, composta de oito sessões, uma vez por semana de TCC, teve início em dezembro de 2005, sendo voltadas para a reestruturação das cognições distorcidas das SC relacionadas aos AP.

Inicialmente, o que a deixava mais apreensiva era ônibus cheio, metrô e túneis longos e escuros. Nessas ocasiões eram comuns sintomas de falta de ar, sensações de desmaio e medo intenso, por temer infarto ou morte iminente.

Nas oito sessões subsequientes, continuamos a reforçar os comportamentos aprendidos com a finalidade de minimizar a apreensão e a ansiedade antecipatória ainda existentes, além do medo de vivenciar determinadas situações. Essas sessões foram voltadas à exposição in vivo da paciente às situações agorafóbicas, em que ela procurou experimentar cada situação, da menos temida a mais causadora de estresse ou ansiedade.

Este estudo foi realizado no Laboratório de Pânico e Respiração do IPUB (UFRJ) e aprovado pelo Comitê de Ética em Pesquisa (CEP - IPUB/UFRJ) dessa instituição, seguindo a orientação da Declaração de Helsinque (1989). A paciente do estudo foi informada sobre a pesquisa e assinou o Termo de Consentimento Livre e Esclarecido.

\section{Resultados}

Após superar o medo das SC por intermédio das técnicas da TCC com exercícios de indução de sintomas, a paciente pôde reunir dados para invalidar suas previsões cognitivas temerosas e passou a contestar os pressupostos temíveis associados a diversas situações. Começou a desenvolver estratégias individuais, como exercícios de relaxamento e respiratórios, para lidar com o TP.

O TP é um transtorno que se desenvolve em indivíduos psicológica e biologicamente vulneráveis (Barlow, 1988), a partir de cognições distorcidas. Uma vez estabelecida a cognição distorcida, esta passará a ser uma referência cognitiva, sendo as respostas e SC subseqüentes resultantes desse conteúdo. Por isso, para se obter sucesso no tratamento e, consequientemente, reduzir as comorbidades, é importante identificar e reestruturar as cognições relacionadas às SC. Enquanto prevalecer o padrão distorcido de pensamento, a resposta produzida será sempre o comportamento desadaptativo que gera 0 AP. Desta forma, tem-se considerado a TCC uma intervenção fundamental na abordagem terapêutica do TP.

Ao final, observamos que apenas a medicação não foi responsável pelo controle dos ataques de pânico. Obtiveram-se os resultados e os critérios para identificar a melhora da paciente na análise dos instrumentos aplicados em dois momentos e que apontaram o controle dos ataques de pânico e a diminuição dos sintomas 
relacionados ao uso da medicação em parceria com as sessões de TCC. Esta última possibilitou uma modificação de distorções cognitivas presentes antes do início do tratamento com essa técnica.

Atualmente, a paciente circula de ônibus normalmente, consegue andar de metrô desacompanhada e atravessa túneis longos e escuros. Houve remissão dos ataques de pânico e extinguiram-se os comportamentos de ansiedade antecipatória e evitação.

\section{Discussão}

No caso apresentado, exercícios controlados de indução dos sintomas foram ministrados em ambiente seguro para que a paciente pudesse entrar em contato com as próprias sensações. Percebendo que as reações corporais emergentes eram inofensivas e resultado dos mecanismos fisiológicos de adaptação, a paciente pôde aceitá-las sem ansiedade limitante. Assim, também pôde resgatar a referência original dos aspectos cognitivos mal-interpretados, e as idéias e medos hipocondríacos, como o de adoecer, começaram a se extinguir, passando a elaborar novas estratégias para lidar com os pensamentos, sintomas físicos e comportamentos, desta forma readquirindo autoconfiança.

Os pacientes com TP relatam bem menos ansiedade durante os procedimentos indutores de sensações físicas semelhantes às que ocorrem durante um ataque de pânico espontâneo, ao perceberem que os exercícios de indução dos sintomas realizados no tratamento são seguros e controláveis (Craske e Barlow, 1988; Sanderson et al., 1989), transpondo-os, desta forma, para situações reais.

Briggs et al. (1993) estudaram a descrição dos últimos AP de 1.108 pacientes com TP, que foram divididos em dois grupos, de acordo com a presença de sintomas respiratórios emergentes. O subtipo respiratório sofria mais AP espontâneos e respondeu melhor à imipramina. Também verificaram que os pacientes com o subtipo não-respiratório apresentavam mais $\mathrm{AP}$ situacionais $\mathrm{e}$ pareciam responder melhor ao alprazolam.

Este caso ilustra bem como pacientes com subtipo respiratório de transtorno de pânico podem ter boa resposta à TCC, após correção do padrão inadequado de respiração e exposição a sintomas respiratórios com os exercícios de hiperventilação, seguidos de correção das cognições corporais distorcidas. São necessários estudos com amostras maiores de pacientes, com finalidade de se verificar se há diferenças de resposta à TCC em diferentes subtipos de TP.

Estão crescendo evidências de que a TCC produz alterações neurofisiológicas no funcionamento do cérebro (Furnmark et al., 2002) semelhantes às produzidas com uso de medicação (Brody et al., 1998) ou essas alterações podem estar nas regiões que diferem daquelas tratadas com as drogas (Furnmark et al., 2002).
Num segundo momento, utilizando as técnicas de TCC, conseguimos interferir em alguns desses mecanismos, como na respiração, que apresenta também um componente voluntário. Por meio de exercícios respiratórios (ER), podemos equilibrar a quantidade necessária de oxigênio e gás carbônico no organismo e restituir seu compasso (Holt e Andrews, 1989).

O conhecimento de procedimentos como ER e de relaxamento muscular (Öst, 1988) se constitui em instrumentos de autoconfiança para o paciente, que passa a ter recursos próprios para lidar com o TP, sabendo que, mesmo sem auxílio de outra pessoa, poderá acalmar-se e diminuir a ansiedade. Além de os pacientes serem ouvidos em suas questões e dificuldades, as sessões de TCC também apresentam um conteúdo didático que ajuda o paciente a lidar com o transtorno e a entender a origem, natureza e componentes do TP.

A paciente apresentou redução de queixas hipocondríacas, quando perdeu o medo das sensações corporais. Barsky e Ahern (2004) demonstraram que pacientes em 12 meses de tratamento em grupo com TCC obtiveram respostas de redução nos escores da hipocondria comparados com as do grupo controle.

As conquistas do paciente tornam-se reforço para novas e mais seguras investidas às situações fóbicas. Os pacientes com TP tratados apenas com medicação apresentam resposta mais lenta, enquanto nos tratamentos associados à TCC observamos maior adesão e colaboração do paciente. Hoje, sabemos que a combinação da TCC com a farmacoterapia produz resultados superiores a essas terapêuticas isoladas (Rush e Hollon, 1991).

As SC relacionadas aos mecanismos fisiológicos de adaptação surgem e desaparecem naturalmente. Ao receber apenas intervenções medicamentosas, o indivíduo se priva de aprender tais princípios. Além disso, atribui o controle às medicações, em detrimento de si mesmo. As atribuições de melhora com medicação são observadas na prevenção de recaídas (Basoglu et al., 1994). Em contrapartida, os tratamentos com TCC no TP obtêm sucesso na supressão de medicações observada no término do tratamento (Otto et al., 1992). Daí a importância da combinação de intervenções medicamentosas com intervenções cognitivo-comportamentais.

\section{Referências}

Angst, J.; Dobler-Mikola, A. - The Zurich Study: V. Anxiety and phobia in young adults. European Archives of Psychiatry and Neurological Sciences 235:171-178, 1985.

Bandelow, B. - Panic and Agoraphobia Scale (PAS). Hogrefe \& Huber Publishers, Seattle, 1999.

Barlow, D.H. - Anxiety and its disorders: the treatment of anxiety and panic. Guilford Press, New York, 1988.

Barsky, A.J.; Ahern, D.K. - Cognitive behavior therapy for hypochondriais. JAMA 291:1464-1470, 2004.

Barsky, A.J.; Fama, J.M.; Bailey, E.D.; Ahern, D.K. - A prospective 4 to 5 year study of DSM-III- R hypochondriais. Arch Gen Psychiatry 55:737-744, 1998.

Basoglu, M.; Marks, I.M.; Kilic, C.; Brewin, C.R.; Swinson, R.P. - Alprazolam and exposure for panic disorder with agoraphobia attribution of im- 
provement to medication predicts subsequent relapse. Brit Journal of Psychiatry 164:652-659, 1994.

Beck, A.T.; Emery, G. - Anxiety disorders: and phobias: a cognitive perspective. Basic Books, New York, pp. 88-92, 1985.

Beck, A.T.; Epstein, N.; Brown, G.; Steer, R.A. - An inventory for measuring clinical anxiety: psychometric properties. J Consult Clin Psychol 56: 893-897, 1988.

Briggs, A.C.; Stretch, D.D.; Brandon, S. - Subtyping of the panic disorder by symptom profile. British Journal of Psychiatry 163:201-209, 1993.

Brody, A.L.; Saxena, S.; Schwartz, J.M. et al. - FDG-PET predictors of response in behavioral therapy and pharmaco therapy in obsessive compulsive disorder. Psychiatry Res 84:1-6, 1998.

Chambless, D.L.; Caputo, G.C.; Bright, P.; Gallagher, R. - Assessment of "fear of fear" in agoraphobics: the Body Sensations Questionnaire and the Agoraphobic Cognitions Questionnaire. Journal of Consulting and Clinical Psychology, 1984.

Clark, D.M.; Salkovskis, P.; Gelder, M.; Koehler, C.; Martin, M.; Anastasiades, P.; Hackmann, A.; Middleton, H.; Jeavons, A. - Tests of a cognitive theory of panic. In: Hand, I.; Wittchen, H. (eds.). Pomic and phobias: treatments and variables affecting course and outcome. Springer-Verlag, Berlin, pp. 71-90, 1988.

Craske, M.G.; Barlow, D.H. - A review of the relationship between panic and avoidance. Clinical Psychology Review 8: 667-685, 1988.

DSM - IV-TR. - Manual Diagnóstico e Estatístico de Transtornos Mentais. 5. ed. Artes Médicas, Porto Alegre, 2000.

Ehlers, A.; Margraf, J. - The psychophysiological model of panic attacks. In: Emmelkamp, P.M.G.; Everaed, W.T.; Kraaimaat, F.W.; Van Son, M.J. (eds.). Fresh perspectives on anxiety disorders. Sweets \& Zeitlinger, Lisse, the Netherlands: pp. 1-29, 1989.

First, M.B.; Spitzer, R.L.; Gibbon, M.; Williams, J.B.M. - Structured Clinical Interview Diagnostic (SCID) for DSM-IV Axis I Disorders-Clinician Version (SCID-CV). American Psychiatric Press, Washington, DC, 1997.

Furnmark, T.; Tillfors, M.; Marteinsdouir, I. et al. - Common changes in cerebral blood flew in patiens with social phobia treated with citalopran or cognitive-behavioral therapy. Arch Gen Psychiatry 59:425-433, 2002.

Goisman, R.M.; Warshaw, M.G.; Peterson, L. G.; Rogers, M.P.; Cuneo, P.; Hunt, M.F.; Tomlin-Albanese, J.M.; Kazin, A.; Gollan, J.K.; Epstein-Kaye,
T.; Reich, J.H.; Keller, M.B. - Panic, agoraphobia, and panic disorder with agoraphobia: data from a 4 percent multicenter anxiety disorders study. Journal of Nervous and mental Disease 182:72-79, 1994.

Holt, P.; Andrews, G. - Hyperventilation and anxiety in panic disorder, agoraphobia, and generalized anxiety disorder. Beh Res and Therapy 27:453-460, 1989.

Lang, A.J.; Craske, M.G. - Pânico e fobia. In: White, J.R.; Freeman, A.S. (eds). Terapia cognitivo-comportamental em grupo para populações e problemas específicos. Roca, São Paulo, pp. 71-107, 2003.

Markowitz, J.S.; Weissman, M.M.; Ouellette, R.; Lish, J.D.; Klerman, G.L. Quality of life in panic disorder. Arch Gen Psychiatry 46: 984-992, 1989.

Marks, I.M.; Mathews, A.M. - Brief standard self-rating for phobic patients. Behav Res Ther 17:263-267, 1979.

Öst, L.G. - Applied relaxation vs. progressive relaxation in the treatment of panic disorder. Behaviour Research and Therapy 26:13-22, 1988.

Otto, M.W.; Pollack, M.H.; Meltzer-Brody, S.; Rosenbaum, J. - Cognitivebehavioral therapy for benzodiazepine discontinuation in panic disorder patiens. Psychopharmacology Bulletin 28:123-130, 1992.

Razran, G. - The observable unconscious and the inferable conscious in current soviet psychophysiology: interoceptive conditioning, semantic conditioning, and orienting reflex. Psychological Review 68:81-147, 1961.

Rice, D.P.; Miller, L.S. - The economic burden of mental disorders. Advances in Health Economics anti Health Services Research 14:37-53, 1993.

Rush, A.J.; Hollon, D. - Depression. In: Beitman, B.D.; Klerman, G.L. (eds.). Integrating pharmacotherapy and psychotherapy. 1. ed. American Psychiatric Press Inc., Washington, D.C., 1991.

Sanderson, W.S.; Rapee, R.M.; Barlow, D.H. - The influence of an illusion of control on panic attacks induced via inhalation of 5.5 percent carbon dioxide enriched air. Arch Gen Psychiatry 48:157-162, 1989.

Wittchen, H.U. - Natural course and spontaneous remissions of untreated anxiety disorder: results of the Munich Follow-up Study (MFS). In: Hand, H.; Wittchen, H. (eds.). Panic anal phobias: treatments and variables affecting course anal outcome. Springer-Verlag, Berlin, pp. 3-17, 1988.

Zaubler, T.S.; Katon, W. - Panic disorder and medical comorbidity: a review of the medical and psychiatric literature. Bulletin of the Menninger Clinic 60 (2, Suppl. A): A12-A38, 1996. 